Sollte die Etatisierung im Nachtragshaushalt nicht zustande kommen, ist der aktuelle Terminplan nicht zu halten, und auch die Alternativlösung mit vorzeitiger Nutzung der Tiefgarage wäre nicht mehr möglich. Allen Beteiligten ist klar, dass jede weitere Verzögerung bzw. Planungspause unweigerlich zu weiteren Kostensteigerungen führen wird.

Die Anmietung eines zweiten Ausweichmagazins wäre, wie immer wieder betont, die unmittelbare Folge, wodurch dem Land Baden-Württemberg jährliche Mehrkosten in Höhe von 2,5 Mio. Euro entstehen würden. Dazu kämen einschneidende
Serviceeinbußen für die Benutzer sowie deutlich schlechtere Arbeitsbedingungen für die Mitarbeiterinnen und Mitarbeiter der WLB.

Angesichts der bisherigen Investitionen des Landes Baden-Württemberg in dieses Bauprojekt sowie der zu erwartenden Mehrkosten, sollten sich noch weitere Verzögerungen ergeben, bleibt nur zu hoffen, dass die Dringlichkeit des Erweiterungsbaus und die Notlage der WLB - immerhin die größte wissenschaftliche Bibliothek des Landes - endlich erkannt und entsprechend gehandelt wird.

\title{
Grenzüberschreitende Bibliotheksarbeit
}

\author{
Die Bibliothekspartnerschaft zwischen der \\ Bibliothèque nationale et universitaire de Strasbourg \\ und der Württembergischen Landesbibliothek
}

\section{Jahre Elysée-Vertrag - Zehn Jahre deutsch- französische Bibliothekspartnerschaften}

Am 22. Januar 1963 unterzeichneten Konrad Adenauer und Charles de Gaulle den Elysée-Vertrag und legten damit die politische, rechtliche, aber auch symbolische Grundlage für die deutschfranzösische Zusammenarbeit der letzten 50 Jahre. Die Zivilgesellschaft nahm in der Ausgestaltung der deutsch-französischen Beziehungen von Anfang an eine Schlüsselrolle ein: Nach Unterzeichnung des Elysée-Vertrags wurden das Deutsch-Französische Jugendwerk ins Leben gerufen und Städtepartnerschaften sowie Partnerschaften zwischen Schulen, Sport- und Musikvereinen, u.ä., im großen Stile gefördert. Mit in der Gegenwart über 2.200 Städtepartnerschaften weisen Deutschland und Frankreich die höchste "Verschwisterungsquote" weltweit auf. ${ }^{1}$ Mit dem Ziel, auch die Bibliothekswelt der beiden Länder enger miteinander zu vernetzen, lancierte das Goethe-Institut Paris (Abteilung Information \& Bibliothek) anlässlich des 40jährigen Jubiläums des Elysée-Vertrags im Jahr 2003 das
Projekt der deutsch-französischen Bibliothekspartnerschaften. ${ }^{2}$ Als Kenner beider Bibliothekswelten war und ist das Goethe-Institut Paris dafür prädestiniert, interessierten Bibliotheken bei der Suche nach einer geeigneten Partnerbibliothek zu helfen und sie bei der Kontaktaufnahme zu unterstützen. Aus diesem Programm, das inzwischen 14 Partnerschaften zwischen deutschen und französischen Bibliotheken umfasst, ist auch die Zusammenarbeit zwischen der Württembergischen Landesbibliothek (WLB) und der Bibliothèque nationale et universitaire de Strasbourg (BNU) hervorgegangen. Wie alle Bibliothekspartnerschaften im Programm des Goethe-Instituts wurde auch sie vor dem Hintergrund einer schon bestehenden Städtepartnerschaft (seit 1962) geschlossen, allerdings mit dem Unterschied, dass sich erstmals zwei rein wissenschaftliche Bibliotheken in nichtstädtischer Trägerschaft zusammengetan haben: Die WLB als größte wissenschaftliche Bibliothek in Baden-Württemberg ist in Trägerschaft des Landes; die BNU, größte Universitätsbibliothek Frankreichs

(1) http://www.dfjw.org/staedtepartnerschaften [29.05.2013]

(2) Vgl. Bibliothekspartnerschaften: Beschreibung und Ziel des Projekts. Online unter http://www.goethe.de/ins/fr/lp/wis/bib/bps/deindex.htm [31.05.13] 
und landesweit an zweiter Stelle nach der Pariser Nationalbibliothek, wird vom Ministerium für Hochschulwesen (ministère de l'Enseignement supérieur et de la Recherche) unterhalten.

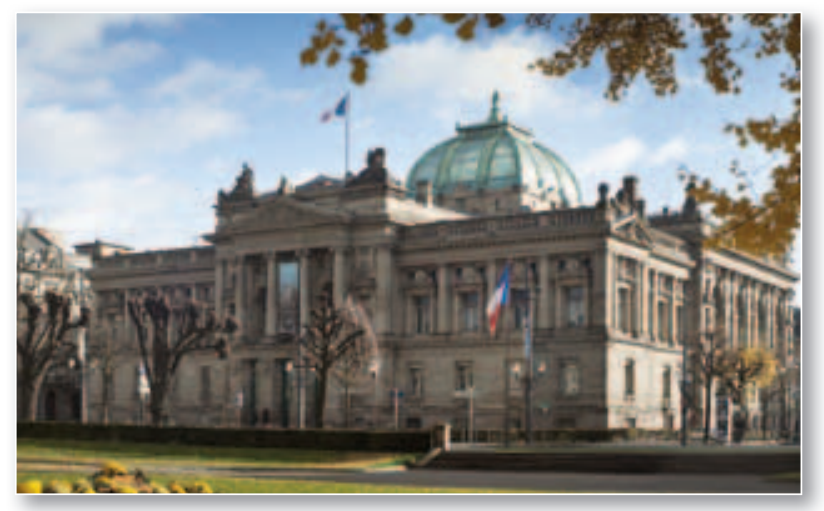

BNU Straßburg

C J JPR-BNU

\section{Ideale Voraussetzungen dies- und jenseits des Rheins}

Ein Blick auf das Profil der beiden Einrichtungen zeigt, dass die Voraussetzungen für eine erfolgreiche Partnerschaft von Anfang an denkbar gut waren: Die WLB wie auch die BNU sind geisteswissenschaftliche Universalbibliotheken mit kostbaren Altbeständen und mehreren bedeutenden Sondersammlungen. Beide Bibliotheken nehmen zum einen landesbibliothekarische Aufgaben (Pflichtexemplar, Landesbibliographie, Sammelschwerpunkt regionale Landeskunde) wahr und sind zum anderen für die subsidiäre Literaturversorgung der Universitäten und Hochschulen im Bereich Geistes- und Sozialwissenschaften zuständig. Als nationale Einrichtung geht der Wirkungskreis der BNU weit über die Stadt Straßburg und die Region Elsass hinaus; so ist sie u.a. Sondersammelgebietsbibliothek und pôle associé der Bibliothèque nationale de France in den Fächern Deutscher Kulturraum und Religionswissenschaften. Die WLB nimmt im Bereich der Zeitgeschichte (ab 1914) eine entscheidende Rolle bei der überregionalen Literaturversorgung ein und kommt mit der Erstellung wichtiger OnlineBibliographien (Landesbibliographie, Internationale Hölderlin-Bibliographie, Stefan George-Bibliographieonline) sowie dem DFG-Handschriftenerschließungszentrum ebenfalls Aufgaben von nationaler Bedeutung nach.

BNU wie auch WLB sind als lebendige Lernorte, aber auch als kulturelle Einrichtungen stark im öffentlichen Bewusstsein verankert und dies, obwohl beide Einrichtungen seit vielen Jahren mit schwie- rigen räumlichen Verhältnissen zu kämpfen haben: Seit Ende 2010 wird das historische Hauptgebäude der BNU an der Place de la République kernsaniert und vollständig umgebaut; die Wiedereröffnung ist für Herbst 2014 vorgesehen. Die WLB hingegen wartet noch auf die Etatisierung eines dringend benötigten Erweiterungsbaus, dessen Eröffnung ursprünglich für 2015 fest zugesagt worden war. Stuttgart und Straßburg sind wichtige politische, wirtschaftliche und kulturelle Zentren diesseits und jenseits des Rheins, und dank schneller TGV-Verbindung können beispielsweise Arbeitstreffen problemlos innerhalb der regulären Arbeitszeiten stattfinden. Nicht nur die geographische, sondern auch die kulturgeschichtliche Nähe des Elsass zu BadenWürttemberg versprach sich zu Recht als Garant für interessante partnerschaftliche Ansätze und vermag vielleicht auch zu erklären, warum Deutsch- bzw. Französischkenntnisse erfreulicherweise bei vielen Kolleginnen und Kollegen vorhanden sind.

Wie das Elsass blickt auch die Bibliothèque nationale et universitaire de Strasbourg auf eine wechselvolle deutsch-französische Vergangenheit zurück. Diese beginnt mit ihrer Gründung im Jahr 1871 als damals Kaiserliche Universitäts- und Landesbibliothek, nachdem die Straßburger Stadtbibliothek mit ihren rund 300.000 wertvollen Bänden im DeutschFranzösischen Krieg durch einen Brand vollständig zerstört worden war. Das Elsass war im Friedensvertrag von 1871 an das Deutsche Reich gefallen, und die Gründung der BNU nach dem Vorbild deutscher Forschungsbibliotheken sollte den schmerzhaften Verlust der Stadtbücherei wieder gutzumachen. Bücherspenden aus der ganzen Welt, darunter 10.000 Dubletten aus der Königlichen Universitätsbibliothek zu Königsberg sowie 4.000 Bände aus der Privatbibliothek von Kaiser Wilhelm I., legten den Grundstock für die neue Bibliothek, die 1895 in einem Prestigebau im wilhelminischen Stil am Straßburger Kaiserplatz (heutige Place de la République) untergebracht wurde. 1926 - inzwischen war das Elsass wieder französisch - erhielt die Bibliothek in Anerkennung ihrer bisherigen Sonderstellung den für Frankreich einmaligen Doppelstatus einer „bibliothèque nationale et universitaire". Während der deutschen Besatzungszeit im Zweiten Weltkrieg übernahm Karl Julius Hartmann, Direktor der SUB Göttingen, die Leitung der BNU. Aus dieser Zeit stammen auch rund 4.100 Dokumente, in erster Linie Landkarten, 
die gegen Ende des Krieges zur Evakuierung nach Göttingen gebracht und erst 2008 zufällig wieder entdeckt worden waren. Die Rückgabe an die BNU erfolgte unlängst im Mai 2013 in einer feierlichen Zeremonie. ${ }^{3}$ Mit ihren wechselnden Zugehörigkeiten und ihren wichtigen deutschen Literaturbeständen sollte die Bibliothèque nationale et universitaire prädestiniert sein für die deutsch-französische Mittlerrolle, die sie in der Gegenwart aktiv ausübt.

\section{Formen der Zusammenarbeit}

Die Bibliothekspartnerschaft, so das Anliegen aller Beteiligten, sollte von einem möglichst breiten Mitarbeiterkreis getragen und mit Inhalten gefüllt werden. Im Jahr 2004 wurden daher in Straßburg wie auch in Stuttgart Präsentationsveranstaltungen zur Partnerbibliothek veranstaltet, die allen interessierten Kolleginnen und Kollegen offen standen. Seither läuft der Informationsfluss über die BNU in der Württembergischen Landesbibliothek vor allem über die regelmäßigen Direktionssitzungen mit dem höheren Dienst und den Sachgebietsleiterinnen und -leitern. Dort werden Themen von Partnerschaftstreffen sowie Neuigkeiten aus der BNU angesprochen und über das Protokoll an alle Mitarbeiterinnen und Mitarbeiter weitergegeben. Zu größeren Ereignissen im Rahmen der Partnerschaft sind darüber hinaus Artikel in der Bibliothekszeitschrift „WLBforum" erschienen.

Seit vielen Jahren verbindet zudem ein offizieller Schriftentausch die Bibliotheken, so dass alle Ausstellungskataloge und sonstige von den Einrichtungen herausgegebene Schriften auch in der Partnerbibliothek verfügbar sind und viele Kolleginnen und Kollegen im Umlaufverfahren erreichen.

Die BNU gibt seit 2010 mit der „Revue de la BNU“ eine Zeitschrift heraus, die mit anspruchsvollen Beiträgen an der Schnittstelle von Bibliothekswesen und den Künsten weit über eine normale Bibliothekszeitschrift hinausgeht. ${ }^{4}$ In dieser Zeitschrift, die für das französische Bibliothekswesen eine Art "Tor zum deutschen Wissen" darstellt, sind auch Beiträge von Kolleginnen der WLB erschienen, und weitere werden gewiss folgen.

Im deutsch-französischen Jahr können die Württembergische Landesbibliothek und die Bibliothèque nationale et universitaire de Strasbourg auf nunmehr zehn Jahre grenzüberschreitende Bibliotheks- und

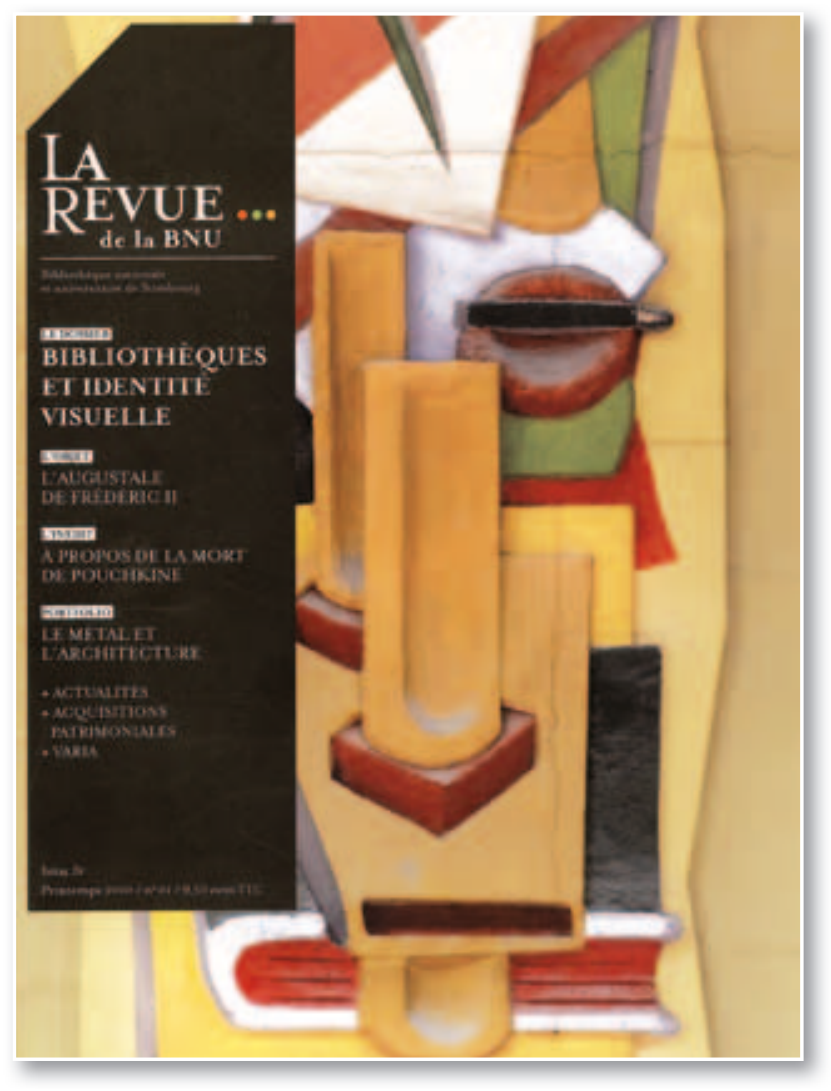

Kulturarbeit zurückblicken. Die Beziehungen zwischen den beiden Einrichtungen beruhen auf den drei Achsen Informationsaustausch, Personalaustausch sowie gemeinsame Veranstaltungsprojekte, die im Folgenden näher vorgestellt werden sollen.

\section{Intensivierung des Informationsaustauschs}

Ein regelmäßiger Informations- und Erfahrungsaustausch bildet das Fundament für die Zusammenarbeit der beiden Bibliotheken: Partnerschaftstreffen auf Direktionsebene finden ein- bis zweimal im Jahr, abwechselnd in Straßburg und in Stuttgart, statt; in Phasen gemeinsamer Projekte kommen weitere Treffen, meist auf Arbeitsebene, hinzu. Bei Partnerschaftstreffen steht grundsätzlich ein „Bericht aus den Häusern" auf der Tagesordnung, bei dem die Direktoren kurz auf aktuelle Entwicklungen und laufende Projekte in ihren Einrichtungen eingehen. Die ersten Jahre der Partnerschaft standen im Zeichen des Kennenlernens, und bei ausgiebigen Hausrundgängen und Besuchen in einzelnen Abteilungen konnten sich die Besucherdelegationen einen guten Eindruck von der Gastgeberbibliothek machen. Dieser Ansatz hat sich als dienlich erwiesen, und so wird fast immer bei den Treffen Zeit für die Vorstellung aktueller Projekte und neuer Dienstleistungen

(3) Vgl. Didier, Christophe (Hrsg.): Von Strassburg nach Göttingen: eine fast vergessene Geschichte. Online verfügbar: http://issuu.com/ bnustrasbourg/docs/gottingen-alld [29.05.2013]

(4) Vgl. http://www.bnu.fr/action-culturelle/la-revue-de-la-bnu [07.08.2013] 
eingeplant. Zahlreiche Themen von Ausleihservices über Digitalisierung bis hin zur Öffentlichkeitsarbeit - und die dafür verantwortlichen Kolleginnen und Kollegen - haben so im Laufe der letzten Jahre die Partnerschaft aktiv mitgestaltet. In manchen Bereichen fand darüber hinaus auch ein vertiefter fachlicher Austausch zwischen den Abteilungen statt, und infolge der Kontakte auf dem Gebiet der Landesbibliographie und der Digitalisierung ist die BNU inzwischen auch Mitglied in den AGs Regionalbibliographie sowie Digitale Bibliothek BadenWürttemberg.

Der Erfahrungsaustausch der vergangenen Jahre stand vielfach im Zeichen des Bibliotheksbaus, und in Anbetracht der Bauprojekte beider Bibliotheken traf es sich gut, dass in den letzten Jahren sowohl in Straßburg als auch in Stuttgart zwei weitere Bibliotheksneubauten entstanden sind: Bei Informationsbesuchen in der neuen Straßburger Médiathèque André Malraux im Juni 2009 sowie in der Stadtbibliothek Stuttgart im November 2009 und - nach Eröffnung des neuen Gebäudes - im März 2011 konnten die Direktoren und ihre Begleiter innovative Raumkonzepte auf sich einwirken lassen und Erfahrungen zu neuesten Standards bei Bibliotheksservices und -automatisierung austauschen. Derartige Besichtigungen sind auch deshalb so wertvoll, weil sie die Begegnung und den Austausch mit anderen Akteuren der jeweiligen Bibliothekslandschaft befördern und damit dem vom Goethe Institut intendierten Ziel des Aufbaus eines "aktiven Netzes von Informationsknotenpunkten" entgegenkommen.

Das Interesse am Bibliothekswesen des Nachbarlands ist groß, und so dreht sich der Erfahrungsaustausch immer wieder auch um bibliothekarische Strukturen und Entwicklungen in Deutschland und Frankreich: Von Bibliotheksstatistik über zentrale bibliothekarische Förderprogramme bis hin zur Vermittlung geeigneter Ansprechpartner dies- und jenseits des Rheins - der Blick ins Nachbarland hat sich oft als anregend erwiesen und so auch die Erfahrungen und Einschätzungen der Kolleginnen und Kollegen der Partnerbibliothek, zu denen sich zum Teil ein richtiges Vertrauensverhältnis gebildet hat. Die Berufung von Dr. Hannsjörg Kowark in den wissenschaftlichen Beirat der BNU sowie den der Revue de la BNU kann hierfür als Beleg angesehen werden.
Gilt die BNU in Frankreich schon seit langem als eine Art Tor zum deutschen Bibliothekswesen und erhält sie daher regelmäßig Anfragen das deutsche Bibliothekswesen betreffend, so war die Württembergische Landesbibliothek in den letzten Jahren auch mehrmals Anlaufpunkt für bibliothekarische Besuchergruppen aus Frankreich, u.a. 2010, als - auf Empfehlung der BNU - eine größere Delegation von Conseillers du livre et de la lecture der WLB einen Informationsbesuch abstattete und sich über Aufgaben und Dienstleistungen der Bibliothek vor dem Hintergrund des deutschen Bibliothekswesens informierte.

\section{Personalaustausch}

Der Personalaustausch stellt seit Beginn der Bibliothekspartnerschaft eine wichtige Säule der Zusammenarbeit dar: Seitens der WLB absolvierten zwei Fachreferentinnen mehrwöchige Praktika in der BNU, und zwei FAMI-Jahrgänge und ihre Ausbildungsverantwortlichen besuchten die Bibliothek zu einem eintägigen Informationsbesuch. Aus dem Kollegium der BNU waren ebenfalls eine Kollegin und ein Kollege des höheren Dienstes zu mehrwöchigen Fachaufenthalten in der WLB: Die Kollegin beschäftigte sich dabei ausführlich mit der Digitalisierung von Zettelkatalogen, während der Kollege Archivbestände für eine Ausstellung sichtete, die beide Bibliotheken wenige Jahre später verbinden sollte. Zwei weitere Kollegen des höheren Dienstes statteten im Jahr 2012 der WLB einen zweitägigen Informationsbesuch ab, in dessen Mittelpunkt vor allem die jeweiligen Arbeitsbereiche (Landesbibliographie, Elektronische Medien, Ausstellungen und Fachreferat) und deren Abläufe an der WLB standen.

2011 kehrte einer der Straßburger "Praktikanten" im Rahmen einer Abordnung an die WLB zurück, um dort eine durch Elternzeit vakante Stelle zu vertreten. Dank seiner Qualifikation, aber auch weil er die WLB durch den Fachaufenthalt und ein gemeinsames Ausstellungsprojekt schon kannte, übernahm der Kollege ohne größere Einarbeitung die frei gewordenen Aufgaben und stellte nebenbei noch die viel beachtete Ausstellung "'Enfance, mon amour'. Die Jugend in der französischen Literatur" auf die Beine. ${ }^{5}$ Seine aktive Mitarbeit in der WLB über acht Monate hinweg hat den Erfahrungs- und Wissensaustausch in starkem Maße befördert und 
die Bibliotheken in diesem Zeitraum auch personell eng zusammenwachsen lassen.

\section{Enfance,}

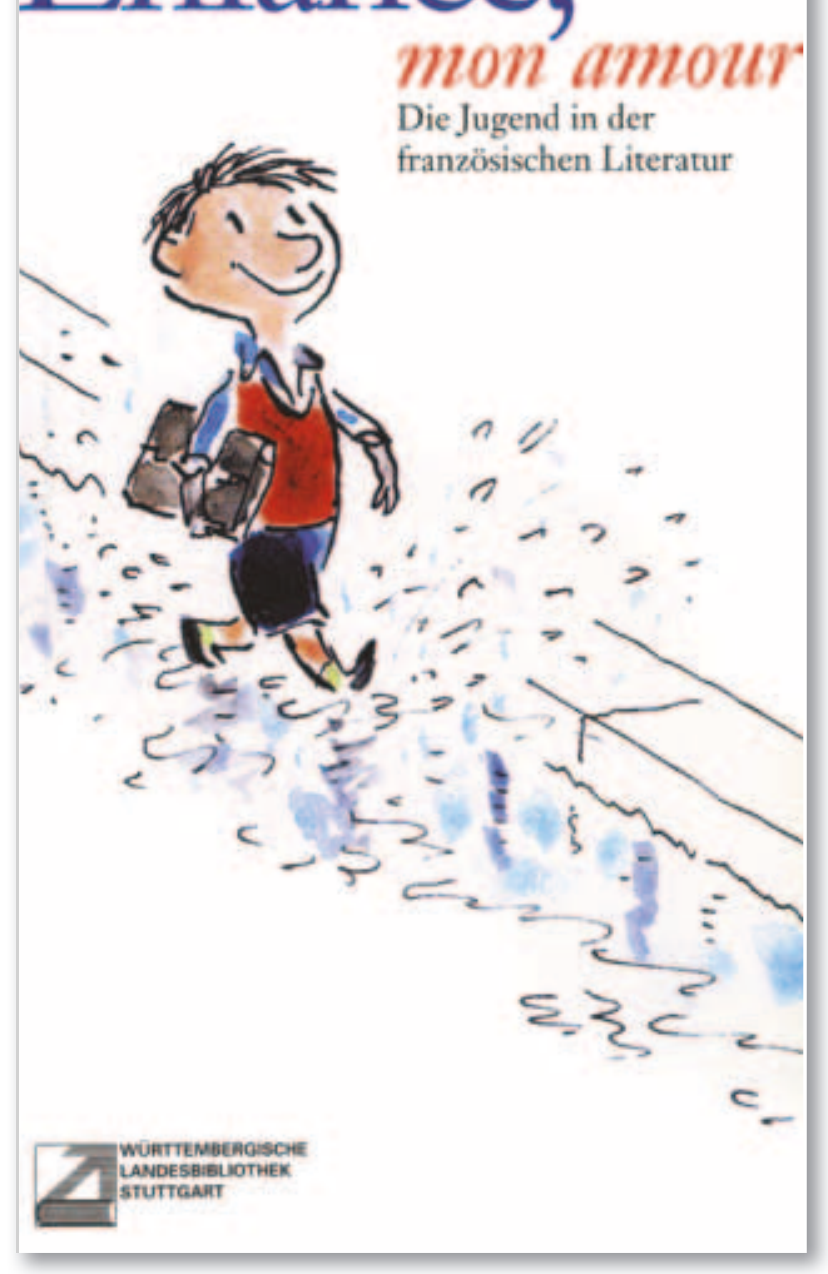

\section{Gemeinsame Veranstaltungstätigkeit}

Seit Bestehen der Kooperation sind die beiden Bibliotheken viermal mit gemeinsamen Ausstellungen an die Öffentlichkeit getreten. Die Zimelienausstellung "Impressions d'Europe. Trésors de la BNUS entre France et Allemagne" aus dem Jahr 2003 bildete gleichsam den Auftakt zur Bibliothekspartnerschaft. Die in Straßburg unter der Ägide von Christophe Didier konzipierte Ausstellung widmete sich den wertvollen Sonderbeständen der Bibliothek, wie z.B. der Ägyptologischen Sammlung mit Papyri und Keilschriften, aber auch zahlreichen Buchbeständen, von denen viele einen ausgeprägten Bezug zu Deutschland, insbesondere auch zu Württemberg, aufweisen, so z.B. die Privatbibliothek Ludwig Uhlands oder die Werke des Tübinger Verlegers Cotta. Teile der sehr umfangreichen Straßburger Ausstellung wurden unter dem Titel "Impressions d'Europe. La Bibliothèque nationale et universi- taire de Strasbourg" ab Oktober 2004 in deutscher Fassung gezeigt, ergänzt um einen Einführungstext zur Geschichte der BNU im Buchmuseum der Württembergischen Landesbibliothek. Bei der Vernissage in Anwesenheit des damaligen Staatsund Europaministers Dr. Christoph Palmer und des französischen Generalkonsuls Dr. Henri Reynaud wurde zwar der Beginn der Bibliothekspartnerschaft offiziell gefeiert, jedoch nicht zeitgleich unterzeichnet. Diverse personelle Wechsel - zuerst in der BNU, wo Albert Poirot im Januar 2006 auf Bernard Falga im Amt des Administrateurs folgte, dann bei den Amtsträgern aus Politik und Wissenschaft, die sich als Schirmherren empfohlen hatten - sowie der Wunsch, die Unterzeichnung in einem feierlichen und zugleich öffentlichen Rahmen zu veranstalten, hatten zur Folge, dass die offizielle Besiegelung der Partnerschaft erst am 12. November 2008 anlässlich der Ausstellungseröffnung von "Orages de papier / In Papiergewittern" in Straßburg stattfand. ${ }^{6}$ Um auch die Stuttgarter Öffentlichkeit an dem Ereignis teilhaben zu lassen, wurden die Urkunde und Fotos von der Unterzeichnung zusammen mit einer Infotafel über die Bibliothèque nationale et universitaire im Foyer der WLB präsentiert.

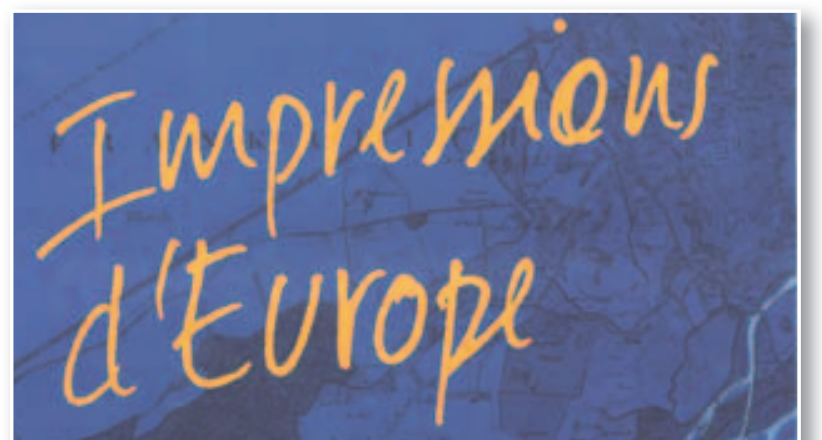

Trésors de la BNUS entre France et Allemagne

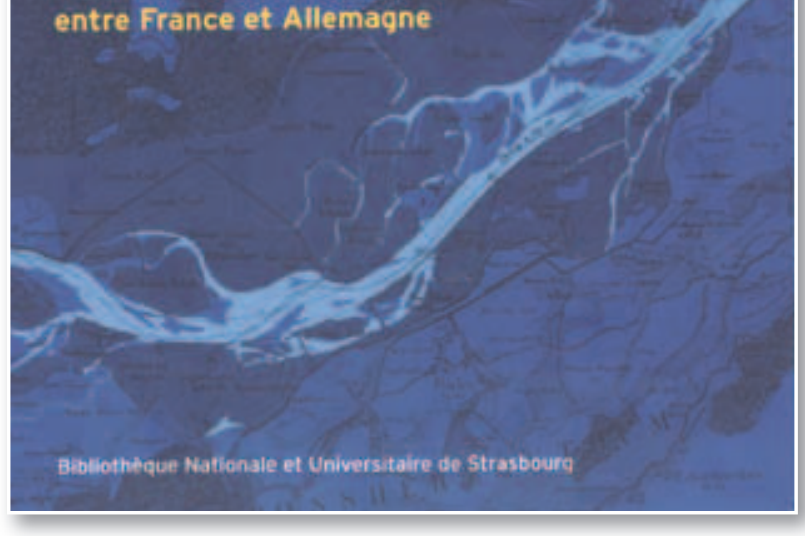

(5) Collonges, Julien: Enfance, mon amour. Die Jugend in der französischen Literatur. Stuttgart: Württembergische Landesbibliothek, 2011.

(6) Neben den Bibliotheksdirektoren Albert Poirot und Dr. Hannsjörg Kowark unterzeichneten als Schirmherren der Bibliothekspartnerschaft: Claire Lovisi, Recteur de l'Académie de Strasbourg, Chancellier des Universités d'Alsace; Dr. Dr. Heinz-Peter Seidel, Generalkonsul der Bundesrepublik Deutschland in Straßburg; Gérard Traband, Vice-Président du Conseil régional D'Alsace und Dr. Veit Steinle, Leiter der Kunstabteilung im Ministerium für Wissenschaft, Forschung und Kunst Baden-Württemberg 
Die zweisprachige Ausstellung „1914-1918. Orages de papier. Les collections de guerre des bibliothèques" und auf Deutsch „In Papiergewittern. Die Kriegssammlungen der Bibliotheken" war ein gemeinsames Ausstellungsprojekt von BNU und WLB, die beide über sog. Kriegssammlungen aus der Zeit des Ersten Weltkriegs verfügen - die BNU als damalige Pflichtexemplarbibliothek für Druckmaterialien über die Westfront und die WLB mit der Bibliothek für Zeitgeschichte (BfZ), einer der größten Spezialbibliotheken für Zeitgeschichte in Europa mit u.a. großen Archivbeständen zu beiden Weltkriegen. Mit der Bibliothèque de documentation internationale contemporaine (BDIC) und der Bibliothèque nationale de France (BNF) schlossen sich im Laufe der Vorbereitungen zwei weitere französische Bibliotheken mit ausgewiesenen Spezialbeständen zum Ersten Weltkrieg dem Projekt an.

Gegenstand der zweisprachigen Ausstellung waren die mediale Darstellung des Ersten Weltkriegs und die durch das Kriegsgeschehen ausgelöste Medienflut. Anhand von Flugblättern, Zeitungen, Plakaten, Fotografien, öffentlichen Bekanntmachungen, u.v.a.m. gelang es den beteiligten Bibliotheken, dieses Phänomen eindrucksvoll darzustellen. An dem gleichnamigen Katalog, der in einer französischen und einer deutschen Version bei Somogy, Paris, erschienen ist, hatten sich neben den Kuratoren aus den Bibliotheken auch mehrere Wissenschaftler aus

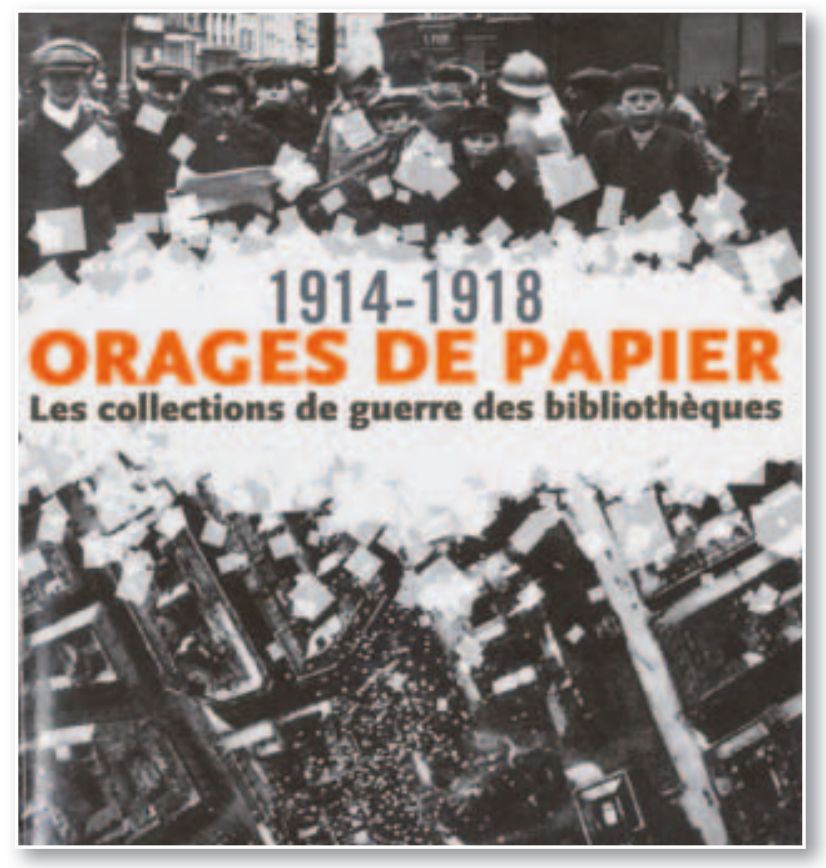

(7) Didier, Christophe (Hrsg.): 1914-1918. In Papiergewittern. Die Kriegssammlungen der Bibliotheken. Paris: Somogy, 2008. Französische Ausgabe: Didier, Christophe (Hrsg.): 1914-1918. Orages de papier. Les collections de guerre des bibliothèques. Paris: Somogy, 2008.

(8) Oelmann, Ute (Hrsg.): Hölderlin - Entdeckungen: Studien zur Rezeption Stuttgart: Württembergische Landesbibliothek, 2008.

(9) Oelmann, Ute u. Therstappen, Aude (Hrsg.): Friedrich Hölderlin. Présences du poète. Paris: Somogy, 2010.
Deutschland und Frankreich beteiligt. ${ }^{7}$ Das Echo in Medien und Wissenschaft war groß und positiv, und die Besucherzahlen in Straßburg, Stuttgart und in Paris, wo die Ausstellung ab Oktober 2010 im Musée d'Histoire contemporaine (Hôtel des Invalides) gezeigt wurde, belegen, dass dieses deutsch-französische Projekt ein breites Publikum erreicht hat.

Nach dem Erfolg von "In Papiergewittern" wurde mit "Friedrich Hölderlin. Présences du poète" kurze Zeit später eine weitere Ausstellungskooperation in die Wege geleitet. Mit dem Hölderlin-Archiv unterhält die Württembergische Landesbibliothek die zentrale Arbeitsstelle für die internationale Forschung über den Dichter Friedrich Hölderlin. Zudem sind rund $80 \%$ der weltweit bekannten Hölderlin-Handschriften im Besitz der WLB bzw. werden dort als Depot verwaltet. 2008 war unter Leitung von Dr. Ute Oelmann an der WLB eine Ausstellung zur Rezeption Hölderlins in den Künsten entstanden. ${ }^{8}$ In Frankreich zählt Hölderlin zusammen mit Schiller zwar zu den am stärksten rezipierten deutschen Dichtern, in der breiten Öffentlichkeit ist er jedoch nur wenig bekannt. Für die Präsentation in Straßburg ab Januar 2010 entstand eine neue, auf das französische Publikum adaptierte Fassung der Stuttgarter Ausstellung, und auch der ins Französische übersetzte Katalog wurde um einige Beiträge renommierter französischer Hölderlin-Forscher erweitert. ${ }^{9}$

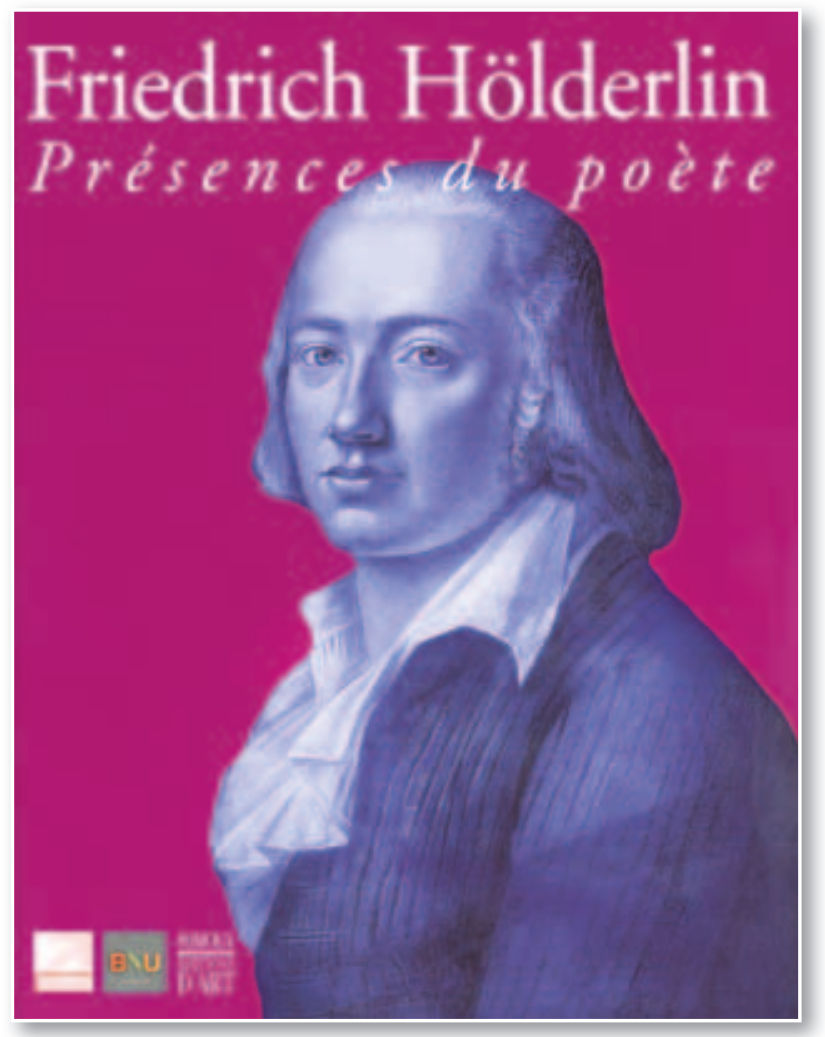


Mit Beginn der Umbauarbeiten an der Place de la République Ende 2010 verfügte die BNU fortan nicht mehr über Räumlichkeiten für Ausstellungen und sonstige Veranstaltungen. Doch dank Kooperationen mit anderen Einrichtungen ist es der Bibliothek gelungen, auch während der Bauzeit ein anspruchsvolles Kulturprogramm aufrechtzuerhalten. Zwei Veranstaltungen sind dabei bis nach Stuttgart gereist: Zum einen die Ausstellung „Drôle d'Europe / Lachendes Europa“, die Europaplakate mit humoristischem Tonfall aus dem Bestand der BNU zeigt und in Zusammenarbeit mit der Badischen Landesbibliothek entstanden war. ${ }^{10}$ Nach ihrem Auftakt 2012 in Karlsruhe wurde die Ausstellung von Juli bis September 2013 in einer erweiterten Fassung in Stuttgart gezeigt.

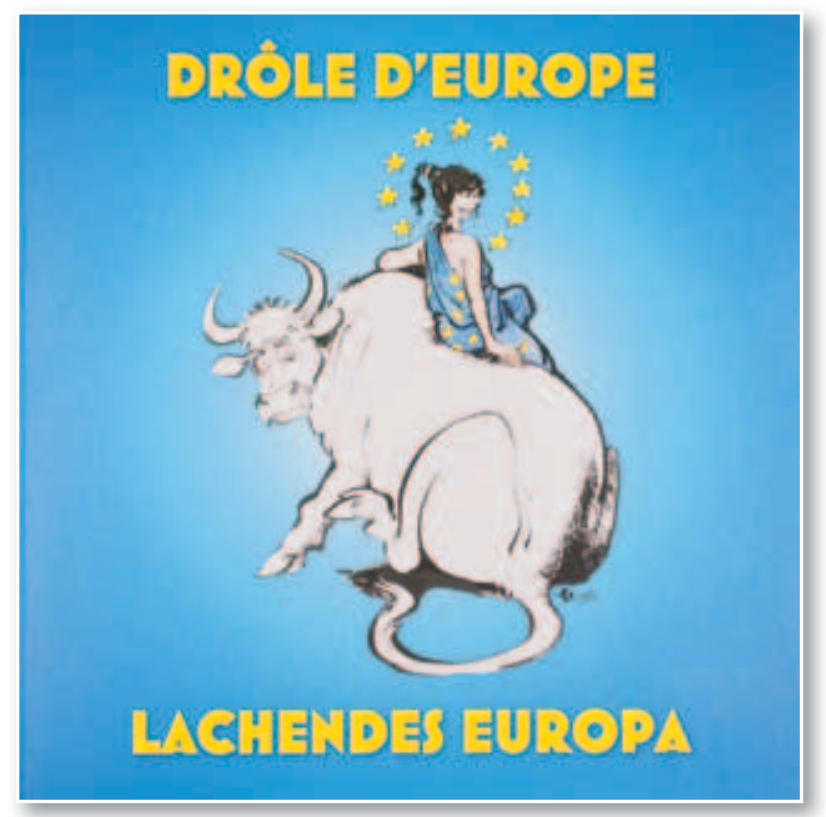

Zum anderen eine szenische Lesung zu René Schickele, die als Kooperationsprojekt von BNU und der Association Culture et Bilinguisme d'Alsace et de Moselle im November 2012 mit großer Resonanz im Straßburger Palais du Rhin uraufgeführt wurde und die im Rahmen der Französischen Wochen im Oktober 2013 in der WLB stattfinden wird.

\section{Ausblick}

Aktuell steht die Gattung Stammbücher im Mittelpunkt der Bibliothekspartnerschaft. In diesen frühen Formen des Poesiealbums, die während der Reformationszeit entstanden und bis zu Beginn des 19. Jahrhunderts vor allem unter Studenten weit verbreitet waren, sind für die heutige Forschung häufig interessante Personen und deren Netzwerke doku- mentiert. Ab Herbst 2013 sollen die Stammbuchsammlungen der beiden Einrichtungen erschlossen, digitalisiert und der interessierten Öffentlichkeit in einer gemeinsamen Internetpräsentation zugänglich gemacht werden. Weitere Straßburger Einrichtungen mit Stammbuchbeständen wollen sich dem Projekt anschließen, das wohl nicht mit der Digitalisierung der Bestände enden, sondern sicherlich eine Fortführung in Form einer Ausstellung und vielleicht auch eines wissenschaftlichen Kolloquiums finden wird.

In Stuttgart blickt man der Neueröffnung der BNU im Herbst 2014 mit Spannung entgegen und vertraut darauf, dass es in den folgenden Jahren weitere Möglichkeiten des Kennenlernens und des Austauschs zwischen Mitarbeiterinnen und Mitarbeitern beider Bibliotheken geben wird. Auch Begegnungen zwischen den Mitgliedern der Freundeskreise von BNU und WLB könnten von großem Reiz sein. Schon 2005 hatten Mitglieder der Württembergischen Bibliotheksgesellschaft die BNU anlässlich der dortigen Ausstellung "Schiller et l'idéal européen" besichtigt und viele interessante und bleibende Eindrücken dabei gewonnen.

In nunmehr zehn Jahren Bibliothekspartnerschaft haben BNU und WLB viele wertvolle Ideen und Impulse aus der Kooperation gewonnen und gemeinsam eine Reihe anspruchsvoller und Aufmerksamkeit erregender Projekte verwirklicht. Wurden beide Bibliotheken in der Vergangenheit vor allem als Informationszentren, Lernorte und Kultureinrichtungen wahrgenommen, so sind sie inzwischen auch Akteure im deutsch-französischen Dialog. Ein Dankeschön gilt den Kolleginnen und Kollegen beider Einrichtungen, die dank ihres Interesses und ihrer Aufgeschlossenheit die Partnerschaft unterstützt und aktiv mitgestaltet haben. Nicht nur aufgrund ihrer reichen, der Öffentlichkeit zum Teil noch wenig bekannten Sonderbestände, sondern gerade auch, weil sie als moderne, sich stets weiterentwickelnde Dienstleistungseinrichtungen auf Austausch angewiesen sind, werden BNU und WLB gewiss auch künftig auf das Potenzial der Bibliothekspartnerschaft setzen und sich immer wieder zu gemeinsamen Projekten zusammenfinden.

Christophe Didier, Birgit Oberhausen

(10) Girold, Elise u. Stello, Annika (Hrsg.): Drôle d'Europe. Lachendes Europa. Karlsruhe: Badische Landesbibliothek, 2012. 